\title{
Ethnologies
}

\section{Le surgissement du terme " africain " pendant la révolution de Saint-Domingue}

\section{Vertus Saint-Louis}

Volume 28, numéro 1, 2006

Haïti - Face au passé

Haïti - Confronting the Past

URI : https://id.erudit.org/iderudit/014153ar

DOI : https://doi.org/10.7202/014153ar

Aller au sommaire du numéro

Éditeur(s)

Association Canadienne d'Ethnologie et de Folklore

ISSN

1481-5974 (imprimé)

1708-0401 (numérique)

Découvrir la revue

Citer cet article

Saint-Louis, V. (2006). Le surgissement du terme « africain " pendant la révolution de Saint-Domingue. Ethnologies, 28(1), 147-171.

https://doi.org/10.7202/014153ar
Résumé de l'article

Deux constats sont à l'origine de cet article : le sens attribué dans la vie courante en Haïti aux termes " nègre ", " africain " et " citoyen ", ensuite la distinction entre citoyens et Africains observée dans certains textes relatifs à l'abolition de l'esclavage en 1793 et 1794. L'auteur relève l'usage du vocable africain par les acteurs qui ont dominé la scène politique sous la révolution haïtienne. Il démontre que leur emploi de ce terme soulève un problème important, relatif au statut social, à l'identité et à la nationalité, pas seulement des anciens esclaves, mais de tous les indigènes. Les chefs mulâtres ne revendiquent aucune origine africaine et désignent les cultivateurs sous le vocable " africain ", considéré comme infamant. Les leaders noirs se taisent sur leur origine africaine et sont aussi impitoyables que les chefs mulâtres envers les cultivateurs. Ces dirigeants perpétuent une image négative de l'Africain. Le courant indigéniste a essayé, au début du XX⿳亠丷厂 siècle, de réhabiliter l'image de l'Africain aux yeux de l'Haïtien, mais sa reconstruction de l'histoire n'est pas scientifique, car il est impossible de faire des leaders noirs de la révolution des représentants des cultivateurs.
Ce document est protégé par la loi sur le droit d'auteur. L'utilisation des services d'Érudit (y compris la reproduction) est assujettie à sa politique d'utilisation que vous pouvez consulter en ligne.

https://apropos.erudit.org/fr/usagers/politique-dutilisation/ 


\title{
LE SURGISSEMENT DU TERME « AFRICAIN » PENDANT LA RÉvolution De SAINT-Domingue
}

\author{
Vertus Saint-Louis \\ École normale supérieure, Port-au-Prince
}

Même si dans les actes de l'état civil, tout Haïtien de 21 ans, maintenant de 18, est un citoyen, dans la pratique, la société admet comme vrai citoyen le propriétaire «demeurant et domicilié » et méritant le titre de «monsieur" parce que marié. À l'extrême opposé se rencontrent ceux qui ne possèdent rien et sont considérés comme errants, vagabonds, sans aveu. Ce sont les " ainsi connus" ou «dénommés» dans les registres des tribunaux, les «individus » dans la société. C'est faire grave injure à un Haïtien que de l'apostropher en créole : «individi sa $\mathrm{a} . .$. nèg sa a » [ce bon à rien, ce nègre]. Vers les années 1950, le terme "africain », peut-être pour cette raison peu employé, véhiculait l'idée d'une injure au sens de vilain, disgracieux, nwè [noir comme du charbon de bois], laid à faire peur. L'usage était assez courant de qualifier une personne de "nèg nwè ».

Depuis, les choses se sont un peu modifiées, mais n'ont pas changé substantiellement. L'idée de la laideur associée à la couleur sombre est vivante dans la pratique, vraiment courante, de l'homme noir «parvenu », surtout à travers la politique, de rechercher une femme de teinte plus claire, soi-disant pour «améliorer la race » par la procréation d'enfants moins foncés. Une telle réalité, vécue au quotidien et dénoncée par le Dr Jean Price-Mars (Price-Mars 1925), porte à s'interroger sur les liens entre la réalité sociale et l'état civil et politique des gens auxquels renvoie le terme « citoyen».

La question se pose depuis l'époque coloniale et pour toutes les catégories sociales. Il suffit pour le constater de jeter un coup d'œil sur 
les registres d'état de la colonie de Saint-Domingue à la veille de 1789, qu'il s'agisse des registres privés de plantation où sont consignés les états des esclaves nés dans la colonie ou importés d'Afrique, ou des registres publics : recensements annuels, état civil (actes de baptême, mariage, décès). Les esclaves y figurent sous les dénominations de nègre, négresses, négrillons, négrites. Il en est de même des libres de couleur, notés comme nègres ou négresses, mulâtres ou mulâtresses, quarterons ou quarteronnes. À partir des années 1760, il est interdit de leur attribuer les titres de Sieur et de Madame. Ils deviennent «le dénommé ", "la dénommée »(Garrigus 1997 : 140). Parmi les blancs, on distingue les propriétaires notés sous la rubrique «hommes », tandis que les petits blancs sont signalés d'après leurs emplois ou métiers, par exemple économe ou raffineur, comme si la qualité d'hommes ne revenait qu'aux riches. Les blanches sont notées comme "femmes", ou parfois « demoiselles » comme si les négresses et mulâtresses n'étaient pas des femmes. Sur les registres privés, le maître distingue l'esclave créole, né dans la colonie, du bossale venu d'Afrique, qui n'est pas signalé sous ce terme mais d'après son ethnie ou "nation » africaine de provenance (Descourtilz 1809, Tome 3 : 117), que l'on nomme nanchon dans la langue créole d'Haïti. Ainsi, l'esclave né en Afrique peut être désigné comme Sénégal, Aradas, Mandingues, Mines, Caplaous, Ibos, Congos, Mondongues, entre autres (Debien 1974).

L'utilisation d'un terme plutôt qu'un autre n'est pas une donnée négligeable. L'esclave accepte le qualificatif de "noir », mais ne souffre pas d'être appelé «nègre » par un blanc. On en a une preuve à l'époque de la proclamation de l'affranchissement des esclaves et des Droits de l'homme et du citoyen à Saint-Domingue. Une dame blanche, veuve d'un fonctionnaire disparu au cours des événements, se voit obligée pour subsister de débiter des victuailles au marché du Cap. Un client ayant oublié de la payer, elle demande à un autre : "Faites-moi la faveur d'appeler ce nègre ». L'homme brandit son sabre en s'adressant à la dame : "Qu'appelles-tu nègre? C'est un citoyen, il n'y a de nègre ici que toi » (Alaux 1935 : 227). La prise en considération de cet incident a déjà donné lieu à un article (Saint-Louis 2000) qui traite des termes «citoyen» et « africain » sous la révolution haïtienne. Cette fois-ci, il s'agira surtout de suivre l'usage du vocable " africain » jusqu'au lendemain de 1804. Cette démarche vise à saisir l'image de l'Africain dont héritera la société haïtienne du début du XIXe siècle, afin de mieux comprendre celle, négative, qui nous est parvenue plus de cent ans après. 


\section{Remplacer " esclave " par " africain "}

En mars 1790, au fort du débat sur les droits politiques de citoyens revendiqués par les libres de couleur, le comte de Behague propose, dans le but de calmer les philosophes, de remplacer par "Africain " le mot " esclave » et d'imiter les Lacédémoniens qui appelaient " hilotes » ceux qu'ils employaient à la culture. En mai 1791, Moreau de SaintMéry ayant proposé que l'Assemblée constituante déclare qu'elle ne se prononcera pas sur l'état des esclaves des colonies, l'incorruptible Robespierre sort de ses gonds pour affirmer :

Le plus grand intérêt... est de rendre un décret qui n'attaque pas d'une manière trop révoltante et les principes et l'honneur de l'Assemblée. Dès le moment où, dans un de vos décrets, vous aurez prononcé le mot : esclaves, vous aurez prononcé et votre propre déshonneur... et le renversement de votre constitution. Eh, périssent vos colonies si vous les conservez à ce prix (Lefebvre 1950:362).

L'Assemblée constituante substitue à « esclave » le vocable "nonlibre » dans le texte de son décret du 13 mai 1791 par lequel elle maintient le statu quo colonial.

On est à la fin de septembre 1791 et, depuis août, les esclaves se sont soulevés dans le Nord de Saint-Domingue. Les libres de couleur de l'Ouest, mobilisant des esclaves, viennent de remporter à Pernier une brillante victoire qui contraint les colons blancs à leur reconnaître la qualité de citoyens. Les colons de Port-au-Prince découvrent, alors, les vertus des esclaves quand il s'agit de faire la guerre. Ils constituent un corps d'esclaves armés baptisés Africains placés aux ordres d'un de ces derniers, dénommé Philibert. Le colon blanc Caradeux, si cruel envers les esclaves, utilise, contre les libres de couleur, ces Africains, dirigés par un de ses fils mulâtres. Les affranchis, dans le Sud, recrutent des esclaves comme force de frappe pour en imposer aux colons. L'insurrection dans le Nord est dominée par des esclaves privilégiés et des libres de couleur, tel Candi, mulâtre, ou Toussaint, noir. Lorsque la première commission civile débarque, en novembre 1791, la plupart des chefs recherchent une entente avec les colons. La métropole adopte la loi du 4 avril 1792 qui admet comme citoyens les affranchis propriétaires et/ou payant taxes. Elle confie la mise à exécution de cette loi à une seconde commission civile, composée de Polvérel, Sonthonax et Ailhaud, qui disposera d'une force armée de 14000 hommes. La loi du 4 avril, parvenue dans la colonie à la fin de 1792, 
semble réaliser la concorde entre colons blancs et libres de couleur, lorsque la deuxième commission civile débarque au Cap, le 20 septembre 1792. Le développement de la contre-révolution, suite à la chute de la monarchie au lendemain du 10 août en France, ranime l'espoir des colons. Ils pensent que la contre-révolution ramènera au pouvoir en France la monarchie qui rétablira l'ancien système colonial. En 1793, les colons continuent d'employer le corps des Africains de Philibert pour terroriser les mulâtres de Port-au-Prince.

L'emploi du terme "africain » révèle la confusion qui règne à l'époque. Il est utilisé au sens d'« esclave » par Sonthonax et Polvérel dans leur proclamation de mai 1793, qui rappelle les dispositions atroces du Code noir contre les marrons. Il remplacera assez souvent le mot « esclave » dans leurs décrets révolutionnaires suivants.

Pour faire face à la situation créée par les colons contrerévolutionnaires et leurs alliés anglais et espagnols en guerre avec la France révolutionnaire, Sonthonax et Polvérel, commissaires chargés d'abord de combattre les esclaves, proclament leur affranchissement. L'acte d'affranchissement des esclaves du Nord, le 29 août 1793, proclame que "les hommes naissent et demeurent libres égaux en droits : voilà, citoyens, l'Évangile de la France... ». En son article 3, il déclare que "tous les ci-devant esclaves iront se faire inscrire, eux, leurs femmes et leurs enfants, à la municipalité du lieu de leur domicile, où ils recevront leurs billets de citoyen français, signé du commissaire civil ». À l'occasion de l'affranchissement des esclaves de l'Ouest, Polvérel ordonne un recensement de toute la population de cette province et précise que «tous citoyens ayant sous leur dépendance des Africains ou des descendants d'Africains... seront tenus d'en faire la déclaration " (proclamation du 10 septembre 1793, cité dans Laurent 1980 : 235-236). La même décision sera prise pour le Sud. Si l'on s'en tient à la proclamation de Polvérel du 31 octobre suivant, ces Africains sont des citoyens. En effet, Polvérel y affirme:

Tous les Africains et Africaines descendants et descendantes d'Africains existant actuellement à Saint-Domingue et dans les îles françaises sous le Vent, ceux qui y naîtront à l'avenir ou qui y aborderont sont déclarés libres et égaux à tous les autres hommes, ils jouiront de tous les droits de citoyens français et de tous les droits énoncés dans ladite Déclaration des droits de l'homme (Proclamation du 31 octobre 1793, article 2, cité dans Revue d'histoire des colonies françaises 1949: 372). 
Sur les registres, les anciens propriétaires sont notés comme les déclarants. Chacun d'eux indique le nombre d'Africains qui travaillent sur sa ou ses habitations à titres divers. Le mot "africain » a été employé, semble-t-il, comme substitut au terme «esclave », jugé probablement indésirable. Tous les anciens esclaves nés ou non dans la colonie passent donc sous la dénomination commune d'Africains, parmi lesquels on distingue les créoles, nés dans la colonie, des autres, nés en Afrique, dont on indique systématiquement l'origine ethnique : Mines, Fons, Caplaous... Une divergence éclate alors à propos du mot «africain ».

\section{Une distinction : citoyen/africain}

Le 10 novembre, le mulâtre Savary, de Saint-Marc, écrit à Sonthonax :

Aujourd'hui... un nombre considérable d'Africains se sont rendus en ville et voulaient absolument piller et incendier. Quelqu'un de votre suite s'était arrêté sur la route et avait dit hautement aux Africains qu'il fallait égorger tous les mulâtres. Cette doctrine prêchée à votre passage et sur les traces de votre voiture, nous seraient (sic) devenus funestes si nous n'avions pas été sur nos gardes. Si nous n'avons pas la protection des délégués de la république pour arrêter les anthropophages que l'envie tourmente, nous serons réduits au plus cruel désespoir (cité dans Ardouin 1958, tome II : 64).

Le 12 novembre, Savary ajoute: «Les Africains à qui nous avons fait du bien ont été trompés et soulevés pour nous égorger» (64). Dans un document du 13 novembre, intitulé «Résistance à l'oppression, les ennemis de Sonthonax déclarent", on lit: "cette conduite de Sonthonax n'a d'autre but que d'enlever à la France la possession de cette colonie puisqu'un pays peuplé exclusivement d'Africains, de noirs, ne peut être une colonie française » (65). L'origine du conflit apparaît dans les explications des propriétaires. Telle habitation «susceptible » d'un grand revenu ne donne rien faute "d'Africains attachés » ou à cause de l'abandon des "Africains ». Les terres demeurent en halliers par absence, disent-ils, d'Africains. Ce à quoi Sonthonax réplique :

Vous avez parler (sic) des complots des affricains, vous qui avez souffert tranquillement aux portes de votre ville un rassemblement liberticide formé de blancs et d'hommes de couleur coalisés pour s'opposer de tous leurs moyens aux principes français et au règne de la parfaite égalité... les affricains étaient épouvantés de vos crimes, est-il étonnant qu'ils aient témoigné leur mécontentement, est-il étonnant qu'ils aient 
frémi de voir leurs droits attaqués et leur indépendance civile menacée, vous vous plaignez, (dites vous) de leur cupidité sans bornes, ainsi vous renversez la signification des mots, vous appellez cupidité sans bornes le désir d'être nourri d'être vêtu et de recevoir un juste salaire de ses peines; et vous qui ne songez qu'à perpétuer votre tyrannie et à convertir en or le prix de leurs sueurs et de leurs travaux vous essayez de vous faire passer pour d'innocentes victimes de leur prétendue inhumanité. Croyez-moi, citoyens, quittez vos opinions insensées, persuadez vous bien que dans le langage de la raison, il n'y a de révoltés que les tyrans, il n'y a de cupidité que celle des maîtres, abjurez vos funestes erreurs, allez vous même au devant des affricains ; réunissez vous sincèrement au peuple de Saint-Domingue, ou tôt ou tard vous disparaîtrez tous de dessus la surface de la terre (Sonthonax, 13 novembre 1793 ).

Dans ce texte, Sonthonax demande aux citoyens de se joindre aux Africains. Cela implique l'idée et la réalité d'une distinction, observée par Polvérel. Lorsque la rumeur se répand d'un soulèvement général des Africains de Port-Salut prévu pour le $1^{\text {er }}$ janvier 1794, Polvérel dénonce :

La crainte d'une insurrection générale des Africains n'est qu'un prétexte imaginé par les officiers municipaux du Port-Salut pour rassembler la plus petite partie des citoyens contre la partie la plus nombreuse. Aucun mouvement des Africains ne justifie la crainte que la municipalité a voulu répandre... Les Africains ne pourraient être tentés de s'insurger que contre les ennemis de la liberté générale... et on ne doit pas craindre qu'ils ne recourent jamais à l'insurrection générale tant qu'ils seront sûrs d'être protégés par la République et ses délégués... La distinction faite par les officiers municipaux entre les citoyens qui sont invités à s'armer et les Africains qu'on exclut du titre de citoyens et contre lesquels la municipalité dirige ses mesures hostiles décèle manifestement la haine pour la liberté générale... C'est par des moyens assez semblables que la municipalité de Léogane est parvenue à égarer les habitants de cette paroisse jusqu'au point de se livrer aux Anglais (Polvérel, ordonnance du 24 décembre 1793).

Les déclarations précédentes de Sonthonax et de Polvérel témoignent d'une distinction qu'on peut relever chez Laveaux quand il écrit dans un même texte : "les citoyens, ci-dessous nommés, feront assembler tous les cultivateurs... D'après le recensement général des cultivateurs et de tous les citoyens actuellement employés dans les camps...» (Laveaux : proclamation du 9 mai 1794). Si Laveaux ne présuppose pas d'incompatibilité entre l'état de citoyen et l'activité de cultivateur, son langage demeure néanmoins l'indice d'une nuance qui n'est pas sans importance sociale et politique. 
L'usage social s'impose même dans les textes officiels, comme par exemple dans cette phrase: "Je suis convenu avec l'Affricain de l'habitation Chantilly qu'il viendrait prendre demain les planches nécessaires à la réparation des godets de la roue du moulin de cette habitation », ou dans ce titre : "Etat des journées que les africains ont employés à la nouvelle prise d'eau de la Ravine du Sud» (Gavanon à Polvérel, $1^{\text {er }}$ mars 1794). Dans la prison du Port-au-Prince, il devient d'usage de préciser la qualité d'Africain de celui qui y entre ou s'y trouve. On peut y voir des relevés de ce genre : "L'affricain Hector pour cause de désobéissance aux ordres de Boyé »; "Guérard aux ordres de la commission civile »; «L'affricain Françoise Bouval.... Par les ordres... de Pierre Marmot, officier de municipalité » («Mouvement de la prison de Port-au-Prince », 10 avril 1794). La condition d'africain ne se définit pas uniquement par l'origine sociale, puisque ce Pierre Marmot est un ancien esclave (Duboÿs s.d. : 148-149). La distinction citoyen/africain est bien caractérisée dans cette lettre à Polvérel.

J'ai fait arrêter et conduire en prison le citoyen Hué, directeur de la poste... Cette manœuvre criminelle a été découverte par un jeune affriquain qui l'a rapporté à un affriquain qui l'a rapporté à un autre et qui sont venus me faire la déclaration (Brunache, 7 mars 1794).

Le commissaire Polvérel ne partage certainement pas l'hostilité des indigènes privilégiés envers les nouveaux libres qu'ils considèrent comme Africains. Mais Polvérel se méfie de tous les nouveaux libres parce qu'ils s'appliquent essentiellement à la culture vivrière dans leurs jardins personnels et négligent la participation aux travaux communs en vue de produire des denrées comme le sucre et le café. De plus, Polvérel se méfie des nouveaux libres qui peuvent être manipulés par la contre-révolution. Ces deux motifs expliquent la déclaration suivante de sa part :

L'affricain Zamore, capitaine de la troupe d'Alaou, vient de se rendre devant nous et d'offrir ses services à la République. Il nous dit qu'il a sous son commandement sur l'habitation Meggy, 16 hommes armés de fusils... Nous vous prions de donner les ordres nécessaires pour qu'une patrouille d'anciens et de nouveaux libres, se transporte sur cette habitation... et se [fasse] remettre les 16 fusils. Il est essentiel pour le bon ordre des campagnes que les affricains sortant soit du camp espagnol soit du camp des révoltés contre la République, ne puissent être armés à moins qu'ils s'incorporent dans la Légion (Polvérel, 16 avril 1794). 
Au fur et à mesure que des indigènes privilégiés passent dans le camp de la contre-révolution, les commissaires se rangent plus fermement du côté des Africains. À propos d'une ordonnance adoptée en 1794 par le commandant Faubert, de Petit-Goâve, Polvérel et Sonthonax essaient de clarifier :

Toute mesure qui tendrait à accorder aux domestiques un degré de plus qu'aux cultivateurs serait une violation du principe d'égalité et... elle donnerait à la classe la plus stérile une prérogative dont la classe la plus précieuse serait privée... La formalité qu'on impose aux maitres et aux domestiques pour distinguer ceux-ci de la classe des cultivateurs, blesse encore la liberté des domestiques... Outre la classe des cultivateurs portionnaires et celle des domestiques, il y a encore les africains propriétaires, les africains pêcheurs, les africains artisans, entrepreneurs, marchands... etc. À quel signe la police militaire les reconnaîtra pour les soustraire et les soumettre à l'arrestation ? (Sonthonax et Polvérel, proclamation du 4 mai 1794)

En juin 1794, les commissaires civils s'embarquent pour la France. L'autorité effective passe aux mains des chefs indigènes: Rigaud (mulâtre) dans le Sud, Beauvais (mulâtre) dans l'Ouest, Louverture (noir) et Villatte (mulâtre) dans le Nord, tous placés sous le commandement du général Laveaux, gouverneur français, résidant au Cap. Depuis 1792, Rigaud et son clan de mulâtres sont en train de faire main basse sur les terres des colons partis, mais il leur manque les bras nécessaires à la mise en valeur du sol. Le 25 septembre 1794, Rigaud, usant du terme «Africain» pour désigner les cultivateurs, affirme:

Il s'est écoulé presque une année depuis que les Africains ont obtenu le bienfait inappréciable de la liberté... Puisque toutes les invitations qui leur ont été faites pour leur propre intérêt sont jusqu'à présent inutiles, le plus grand nombre d'entre eux persiste à refuser du travail nous nous voyons forcés de recourir aux mesures d'autorité et de corroborer pour ainsi dire leur bonheur malgré eux-mêmes (Rigaud, ordonnance du 25 septembre 1794).

Pour Rigaud, les Africains sont les cultivateurs. Ils refusent de comprendre qu'ils doivent du travail à la République dont ils ont reçu le bienfait de la liberté. Il décide que tous les Africains attachés aux habitations avant la proclamation générale doivent être contraints d'y revenir et de s'y fixer. Un document du 10 vendémiaire an IV relève, dans la commune des Cayes, trente-sept sucreries ayant chacune un gérant identifié et en tout, 2152 "affriquains » portionnaires (métayers), 1711 «affriquaines idem »; sur vingt-quatre caféières, 
seulement neuf gérants sont identifiés et il s'y trouve 175 «affriquains portionnaires » et 150 «affriquaines idem »; sur huit de ces caféières, il n'y a ni « affriquains » ni « affriquaines » (Gavanon, 10 vendémiaire an IV). En 1797, Rigaud précise: "Quand je dis Africains, j'entends les cultivateurs en général parce qu'ils sont tous ou Africains ou descendants d'Africains " (Rigaud, 18 thermidor an V).

Pendant ce temps, la Révolution aux colonies pose un grave problème de définition d'identité et de nationalité dans laquelle le terme « africain » est directement impliqué.

\section{Un Africain peut-il être Français ?}

Avant la révolution, suivant l'article 57 du Code noir, l'affranchi est sujet à part entière du royaume, la lettre d'affranchissement lui tenant lieu de certificat de naissance même s'il est né en territoire étranger. L'esclave n'existe pas au regard du droit français, mais simplement d'après la législation faite pour les colonies. On pourrait dire « esclave n'est pas français ». Émilien Petit avait certainement établi une distinction importante en rédigeant le Droit public ou Gouvernement des colonies françaises d'après les lois faites pour ces pays (1771) ainsi que le Traité sur le gouvernement des esclaves (1777). Les actes d'affranchissement de Polvérel et de Sonthonax, ainsi que le décret d'abolition de l'esclavage du 4 février 1794 ne dépassent certainement pas le Code noir quand ils font des anciens esclaves des Français. Mais, de même que l'administration royale et l'opinion coloniale ont remis en question, au XVIII ${ }^{e}$ siècle, ce qui a été admis dans le Code noir, de même des représentants de France sous la Convention thermidorienne et sous le Directoire vont contester les droits reconnus en 1793-1794. L'un de ces droits, c'est la nationalité française des indigènes des colonies. En une protestation écrite adressée le 4 février 1794 par les colons au Comité de salut public et à la Convention, ils signalent que Belley, l'un des députés de Saint-Domingue, n'est pas Français, mais « de nation afriquaine-bambara »(Bénot 1993: 17).

La question prend une ampleur politique nouvelle avec l'ascension politique de Toussaint Louverture, chef noir d'esclaves insurgés dans le Nord, entré au service de l'Espagne en juillet 1793 et passé dans le camp de la France aux environs de mai 1794. Les Espagnols vaincus en Europe et à Saint-Domingue signent, en juillet 1795, le traité de Bâle par lequel ils font la paix avec la France. Ils se retirent de la partie Nord 
de Saint-Domingue qui reste aux mains de Toussaint. Un conflit grandissant est en train d'opposer les noirs et les mulâtres de la ville du Cap et, le 22 septembre 1794, Laveaux annonce au gouvernement que " les mulâtres veulent commander, les Africains en nombre immense dans la ville détestent et les blancs et les mulâtres » ("Message à la Convention nationale », $1^{\text {er }}$ vendémiaire an III). Toussaint Louverture réalise une véritable percée politique lorsque, en mars 1796, il vole au secours du gouverneur Laveaux menacé d'être dépouillé de son autorité par les mulâtres de la ville du Cap alliés à ceux du reste de la colonie. Sonthonax, de retour en mars 1796, s'appuie sur Toussaint pour combattre Rigaud. Dans le cadre de la lutte contre les mulâtres du Sud, Sonthonax y envoie une délégation en 1796. Contre Rigaud, la délégation observe :

La qualité d'Africains qu'on leur donnait était un titre d'exclusion aux places. Quelques-uns avaient été élevés au grade de capitaines et, ceux-là, les mulâtres les appelaient officiers de fortune (Kerverseau et Leborgne 1796).

Un ordre du pouvoir central dira :

Il est défendu sous peine d'emprisonnement de désigner soit verbalement ou dans des actes publics et privés les cultivateurs et tous les citoyens noirs des deux sexes sous la dénomination d'africains qui rappellent l'esclavage et forment une distinction particulière. Sont citoyens français et déclarés tels par la loi tous ceux qui habitent le territoire de la République et Saint-Domingue étant partie intégrante de la France, les citoyens noirs qui habitent Saint-Domingue sont Français et non Africains (Kerverseau et Leborgne 1796).

Dans le rapport que Sonthonax adresse au Directoire pour rendre compte de sa gestion des suites de la conspiration des Mulâtres contre Laveaux, il rend hommage aux officiers noirs du Cap et aux Africains qu'ils commandent. Le terme africain désigne, une fois de plus, les cultivateurs. C'est en ce sens que Rochambeau l'emploie lorsqu'il écrit que « les Africains aiment mieux élever les volailles et récolter les vivres du pays parce qu'ils ne partagent pas les bénéfices de ces objets avec les propriétaires »(Rochambeau 1796). Si Rochambeau est déjà quelque peu hostile à ces Africains, il ne fait pas de doute que Sonthonax ne l'est pas du tout et les considère comme des citoyens. Il s'appuie en général sur les Noirs. En mai 1796, il nomme Toussaint général de division puis, en mai 1797, général en chef de l'armée de Saint- 
Domingue. La nouvelle politique de Sonthonax aggrave un conflit déjà en cours.

Lors d'un débat de la Convention, au cours de l'été 1795, les actes d'affranchissement sont vus comme un "triomphe des Africains " (Gauthier 1992: 270). Le représentant Serres tient des propos que Le Moniteur (29 juillet 1795) n'ose reproduire, mais qui provoquent la réplique indignée d'un député : «Est-ce que je suis un chien... Non, reprend Serres, mais tu n'es pas Français » (Wanquet 1998: 244). Le mépris de Serres ne fait pas de distinction de couleur et de classe quand il s'agit des indigènes des colonies. Donc le droit des Africains se pose d'abord en termes de relations internationales entre peuples. En 17961797, pendant les débats aux Conseils sous le Directoire jusqu'au coup d'État du 18 fructidor, si les colons et leurs représentants protègent le mulâtre Villatte et ménagent son collègue Rigaud, c'est pour mieux cibler le républicain Sonthonax et le général noir Louverture. En ce qui concerne les cultivateurs, les colons ne se sentent pas en mesure de demander leur rétablissement pur et simple dans la servitude. Ils exigent qu'on les place dans un état intermédiaire, plus proche de l'esclavage que de la liberté. Selon les colons, les Africains «n'entendent ni le français ni la raison » (Courrier de la France et des colonies, 17 octobre 1795). Le coup d'État du 18 fructidor rend pour un moment le pouvoir aux républicains de France. Les députés de Saint-Domingue, élus en 1796 et 1797, d'abord contestés en France, sont autorisés à siéger aux Conseils. L'un d'eux, Laveaux, ancien gouverneur, est admis au Conseil des Anciens, où il se dresse contre les colons.

Vous convenez que les habitants de l'Afrique étaient les seuls capables de supporter les travaux de défrichement et de la culture. N'est ce pas convenir que c'est à ces mêmes habitants de l'Afrique que vous êtes redevables de vos propriétés foncières, de vos établissements d'agriculture, par suite, de votre commerce. Sans leurs travaux eussiezvous fait des sucres, des cafés, des cotons, de l'indigo. Sans leurs travaux eussiez vous édifié une marine marchande? Ils ont donc fait pour la France plus que tous les autres Français qui ont été s'établir aux îles. Quoi, vous auriez, durant tout le temps de l'esclavage, fertilisé vos sucreries par les sueurs et les fatigues de ces hommes noirs que vous avez ravis à leurs familles, à leurs pères et mères, à leurs habitudes, à leurs épouses! Quoi, vous auriez engraissé vos champs des cadavres de leurs prédécesseurs et aujourd'hui que la loi vous donne le titre de citoyens vous voulez les considérer comme étrangers! (Laveaux 1798) 
Écartant la distinction qu'on prétend établir entre le guerrier et l'homme des champs il avance:

On laisserait croire que tous les citoyens noirs ne sont pas citoyens français. Le cultivateur ne s'est-il pas rendu aussi utile que le noir qui a porté les armes. Avec quoi aurait-on alimenté l'armée si pas un n'eût voulu cultiver la terre? (Laveaux 1798)

C'est à cette époque que Toussaint Louverture, ancien ami, protégé et protecteur de Laveaux, commence à recueillir sa succession au gouvernement de Saint-Domingue. Optera-t-il pour la politique recommandée par Laveaux? Ou bien fera-t-il sien le point de vue des mulâtres, qui se ramène à celui des colons contre-révolutionnaires ? Il est important de répondre à cette question, car les indigénistes haïtiens, essayant de récupérer le passé pour le faire servir à leurs préoccupations politiques de pouvoir, présentent les chefs noirs de la révolution, groupés autour de Toussaint, comme les leaders naturels de la grande masse des esclaves, les représentants authentiques de l'Afrique (Duvalier et Lorimer 1968 : 324). Pourtant, dans le Nord passé sous la férule des chefs noirs Louverture, Dessalines et Christophe, il se produit une équivalence entre «cultivateur» et « africain».

\section{Toussaint Louverture et l'équivalence cultivateur/africain}

La province du Nord est la base de départ de l'ascension politique de Toussaint Louverture, mais c'est en mai 1797 qu'il en obtient le contrôle total sur le plan militaire. Jusqu'à cette date, les commandants militaires locaux y agissent plus ou moins à leur gré. On n'utilise pas pour désigner les cultivateurs le terme «africain». Les vocables « Congo » ou « nègres de Guinée » sont largement employés dès 1795 sur certaines habitations de la région du Nord-ouest (Debien 1962). La plupart des insurrections dites « des Congos » qui éclatent dans le Nordest, en 1796, sont venues de la crainte du rétablissement de l'esclavage par Laveaux, Toussaint ou Sonthonax, qui veulent revenir au système de plantation coloniale (Geggus 1982 : 203). En 1797, les militaires du parti de Toussaint Louverture commencent comme ceux de Rigaud à entrer en possession des terres. La politique de Toussaint envers les cultivateurs est encore libérale. Il a d'autres préoccupations. La montée en puissance des colons dans les Conseils des Cinq Cents et des Anciens en France inspire à Toussaint des craintes pour son avenir personnel et pour la liberté générale. Il expulse le commissaire Sonthonax, 
représentant de la France, un républicain révoqué et rappelé par la métropole à la demande des colons. Lorsque les Anglais se retirent en mars 1798, il entame un processus d'alliance avec les colons, les Anglais et les Américains. Il se procure des ressources qui lui permettent de remporter, au cours d'une longue guerre civile (juin 1799-août 1800), la victoire sur Rigaud, resté nominalement fidèle à la France, dont il attend une aide qui ne viendra pas. Durant cette période, les hommes du parti de Toussaint entrent en possession de nombreuses propriétés de colons. Leur politique à l'égard des cultivateurs, assez modérée jusqu'en 1798, tend, depuis lors, à être identique à celle de Rigaud.

Il est remarquable que l'on ne trouve pas dans les écrits de Toussaint les termes «Africain » et «Congos» pour désigner les cultivateurs. Toussaint ne fait aucune référence à la distinction entre créole et bossale. Il ne connaît que des cultivateurs. L'introduction de l'article 17 de sa constitution ne mentionne pas d'Africains comme dit Madiou, mais des « cultivateurs indispensables au rétablissement et à l'accroissement des cultures». Sa pensée quant à leur statut est exprimée dans l'ordonnance du 12 octobre 1800. Toussaint y prévoit la militarisation de l'organisation du travail. Les cultivateurs obéissent aux conducteurs d'habitations comme les soldats aux sous-officiers et officiers. Les habitations sont soumises aux commandants militaires, eux-mêmes placés sous les ordres des généraux comme Dessalines, nommés inspecteurs de culture. Les cultivateurs n'ont pas le droit de se déplacer sans permission, même pour se rendre au marché. Les esclaves affranchis en 1793 doivent revenir sur les habitations de leurs anciens maîtres pour travailler comme métayers à la production des denrées. Ceux qui résident dans les villes sont traînés de force par l'armée dans les campagnes. Les noirs et mulâtres qui occupent des postes importants dans l'administration sous Toussaint Louverture n'ont rien à voir avec ces cultivateurs et avec l'Afrique. Ils « parlent de tout dans leurs cercles sauf de leur ancien état. Ils proclament avec enthousiasme le nom de la contrée où le sort les a transplantés »(Justin 1826: 326). Selon les chefs indigènes, ce cultivateur est un paresseux, qui ne peut travailler que sous la contrainte. Dessalines affirme : «Nèg pas connoit travay si vous pa forcé yo [Le noir ne travaille que sous l'effet de la contrainte physique]» (Duboÿs s.d., II : 104). Comme Rigaud, Toussaint estime que le cultivateur ne comprend pas la liberté et qu'il faut contribuer à son bonheur malgré lui en lui imposant le travail de la terre. 
En voulant attacher les anciens esclaves au sol, la nouvelle aristocratie indigène maintient un aspect essentiel du droit colonial, énoncé dans le passage suivant.

C'est donc aussi un abus qu'on a estimé par tête les esclaves des habitations qui ont été vendus et de celles évaluées pour fixer la portion revenant aux héritiers. On aurait dû se borner dans l'un et l'autre cas à donner une estimation de la terre en observant qu'il s'y trouvait attaché tel nombre d'individus (Lattre 1805 : 190).

Est-ce de cette vision que dérive le terme «individi » de la langue créole ? Ce n'est pas le moment de répondre à une telle question. Contentons nous de signaler l'erreur profonde de Toussaint et de ses hommes. Depuis 1799, les Américains, qui profitent du commerce de Saint-Domingue, estiment que les Noirs maintenus dans l'agriculture sans se livrer au commerce maritime, comme le veut Toussaint, ne donneront rien (Logan 1940 : 83-84, 125-126).

Dans l'état-major du parti de Toussaint, l'on retrouve des Blancs, des Mulâtres, des Noirs créoles ou nés en Afrique. La politique de Toussaint procède d'un esprit aristocratique qui est celui des Noirs libres, qui étaient principalement tournés, avant 1789, vers l'acquisition de terres et de grades militaires, activités relativement conservatrices par rapport aux risques et exigences du commerce maritime. De ce point de vue, la naissance en Afrique n'est pas un critère décisif. Pierre Augustin et Jean-Baptiste L'Éveillé, dit Riché, futur président d'Haïti, sont des Noirs libres, privilégiés au temps de la colonie, qui deviendront officiers de Toussaint Louverture. Le premier se soucie particulièrement de ne pas évoquer son origine africaine (King 2001: 147, 161). Tous deux suivent la même ligne de conduite que Toussaint, Noir créole affranchi après 1770, ancien propriétaire d'esclaves (Ménier 1976 ; Debien 1977 ; Geggus 1997 : 266-284). Celui-ci n'entend pas qu'on le prenne pour un «nègre de la côte » (Barthélemy 1989: 183), affecte parfois de s'exprimer en latin (Madiou 1989) et ne veut pas qu'un étranger lui parle créole, langue dans laquelle il s'adresse au peuple (Descourtilz 1809, III : 245-246, 251). Toussaint au pouvoir est un parfait aristocrate qui adopte totalement l'idéologie de l'ancien régime colonial. Il a deux cabinets pour recevoir les femmes. L'un, plus ouvert, est destiné aux dames de couleur qu'il qualifie de citoyennes. Un autre, plus particulier, est réservé aux blanches qu'il appelle "madame », leur donnant ainsi priorité par un titre propre à l'Ancien Régime. Louverture reproduit ainsi la conduite des colons blancs hostiles à la liberté générale et dénoncés par Laveaux en ces termes: 
Les exemples les plus frappants ne peuvent les corriger... On ne veut et on ne peut se faire, à voir les nègres libres, à voir cette égalité. Pour qu'elle ne soit pas si apparente, ces hommes, sottement orgueilleux, appellent les nègres citoyens, et entre eux s'appellent Monsieur (cité par Pluchon 1989: 76).

Or, vers la même époque, Lazare Carnot, dans une lettre au ministre de la Marine, lui laisse entendre qu'il est « injuste d'employer monsieur à la place de citoyen » (Carnot 1796). Ainsi, Toussaint applique envers les cultivateurs la politique recommandée par les chefs mulâtres et par les colons contre-révolutionnaires. L'hostilité des cultivateurs ne tarde pas à se manifester.

Après l'expulsion de Sonthonax par Toussaint, en août 1797, une correspondance signale que

Larose à la tête d'une bande... assiège le Port-de-Paix. On le dit soutenu par les Congos de Jean-Rabel dont les noirs ont en général refusé de marcher sous les ordres de Louverture contre le Mirebalais et SaintMarc... Depuis le départ de Sonthonax, les nègres mécontents abandonnent le peu de travail qu'ils faisaient encore. Ils voient dans ce coquin de Toussaint un ambitieux qui a sacrifié l'homme qui était plus nègre que Toussaint. Celui-ci se serait déjà perdu s'il n'avait eu la précaution de s'entourer de cinq à six régiments pour lesquels il sacrifie tout ; tout est à eux, tout leur est permis. Cette force armée est d'autant plus redoutable pour les ateliers qu'elle est dirigée par les mulâtres les plus éclairés et les plus scélérats tels que Raimond. Mais comme le pillage devient leur unique ressource, il s'ensuit que le petit reste de la culture est abandonné et qu'en totalité ils cherchent à se soustraire à la tyrannie de Toussaint (cité par Debien 1962 : 146).

On doit se méfier de ce texte qui émane d'un colon. Il témoigne, toutefois, d'une tension qui ne cessera d'augmenter. L'opposition des cultivateurs à la politique de Toussaint ne cesse de grandir. Lors des manifestations devant le Cap contre Roume, agent de la France, organisées, en 1800, par Louverture, les cultivateurs ont présenté des revendications significatives d'un désaccord radical avec sa politique. Ils se sont plaints que le «ministre du culte refuse d'enterrer les citoyens pauvres... » Une de leurs accusations contre Roume peut bien s'adresser à Louverture. Ils reprochent à l'agent de la France «d'avoir permis aux émigrés de toutes couleurs de rentrer dans la colonie et de les avoir envoyés en possession de leurs biens séquestrés ». Ils demandent que Roume donne aux cultivateurs la propriété de la moitié des terres sans laquelle la liberté n'est qu'un bienfait illusoire. «Ils [déclarent qu'ils] 
sont las d'épuiser leurs forces pour d'ingrats et orgueilleux propriétaires » (cité par Kerverseau 1939: 320-321).

Descourtilz, partisan de l'ancien ordre de choses, observe que

l'impiété qui existe parmi les nègres depuis la révolution a été la cause de désordres, de malheurs réitérés, de forfaits inouïs. Toussaint Louverture croyant ramener les nègres à leurs devoirs, avait ordonné sur chaque habitation une prière du soir. C'est à cette époque que nos nègres divisés par les opinions élevèrent un schisme entre eux. Ils riaient les uns des autres dans les cérémonies pieuses, jusque là de mauvaises mères disaient à leurs enfants de ne pas prier Dieu puisqu'il ne les empêchait pas de mourir (Descourtilz 1809, III : 280, 281).

Il n'est pas question de prendre ce texte pour argent comptant. On ne peut pas non plus lui dénuer toute signification. Ce passage venant d'un contemporain est un indice d'une opposition qui va culminer en insurrection.

L'application de l'ordonnance du 12 octobre 1800 provoque, un an après, un soulèvement des cultivateurs réprimé dans le sang. À cette occasion, Toussaint Louverture promulgue les ordonnances des 10 et 25 novembre 1801 . Il y déclare que

la liberté ne consiste pas à faire ce qu'on veut mais ce à quoi l'on est destiné ; sans quoi la licence prend la place de la liberté. Pour prévenir les maux qui pourraient résulter de cette licence, il est de la sagesse du gouvernement de rendre chacun à son état et de l'obliger à s'en occuper essentiellement.

Toussaint impose comme condition pour résider dans les villes et bourgs la détention d'une carte de citoyenneté à laquelle les cultivateurs n'ont pas droit. Il leur assigne l'habitation comme lieu de résidence obligatoire. Les commandants militaires doivent faire un relevé des cultivateurs, qui sont condamnés à garder leur statut de père en fils. Lors de l'application de ces dispositions à Port-au-Prince, des gens exhibent leurs lettres d'affranchissement d'avant 1793, ce qui fait parler de deux libertés. Dessalines embarrassé réplique, sans convaincre personne, qu'il n'existe qu'une seule liberté (Duboÿs s.d., II : 227).

Entre temps, le premier consul Bonaparte, au pouvoir en France depuis novembre 1799, organise une expédition militaire de 22000 hommes qu'il confie à son beau-frère, Leclerc, avec ordre de rétablir l'autorité de la France et l'ancien régime colonial esclavagiste. Les 
mulâtres comme Rigaud et Pétion, qu'il emploie en tant qu'ennemis de Toussaint, sont simplement des «Africains dorés ». Ils seront utilisés, suivant les instructions de Bonaparte, comme instruments contre Toussaint. Si ce dernier ne résiste pas, ils seront déportés à Madagascar. À ce moment, la politique de ségrégation envers les cultivateurs entraîne des conséquences néfastes pour Louverture. Privé de l'appui en masse et décidé des cultivateurs, il ne peut, en dépit de son génie militaire, résister aux troupes de Bonaparte. Il se transporte au Cap, le 6 mai 1802, pour se soumettre à Leclerc, et demande à Dessalines et Belair, les deux derniers généraux qui lui soient restés fidèles, de faire de même. Des fils obscurs de l'Afrique refusent de déposer les armes.

\section{Résurgence du vocable " africain ”}

En 1793, lorsqu'une fraction importante des indigènes privilégiés rallie les rangs de la contre-révolution, les anciens esclaves, en dépit d'une répression sévère, se soulèvent en masse et gagnent les montagnes dans les régions de Léogane et de l'Artibonite, pour y défendre les droits que la République vient de leur reconnaître. Au lendemain de la soumission de Toussaint, des officiers subalternes de son armée, tels les Macaya, Sans-Souci et autres, poursuivent la résistance en organisant l'insurrection des cultivateurs du Nord. Ils entreprennent une héroïque guérilla et mettent en déroute les forces combinées des troupes indigènes et européennes. En octobre 1802, les anciens officiers de Toussaint et de Rigaud abandonnent le camp français pour échapper à une mort certaine. Ils craignent moins les troupes de la métropole que celles des cultivateurs en armes du colonel Sans-Souci. Christophe invite ce dernier à une rencontre pour discuter de la lutte contre les Français. $\mathrm{Au}$ rendez-vous convenu, il fait assassiner Sans-Souci et la plupart de ses lieutenants. Rien ne prouve que tous les membres du parti de SansSouci soient nés en Afrique. On y rencontre même deux mulâtres. Il est cependant qualifié d'Africain à cause de l'influence des cultivateurs et des chefs militaires estimés d'origine congolaise et incontrôlables.

L'on ne saurait s'arrêter aux seuls facteurs internes comme éléments d'explication. Lorsque les officiers supérieurs des anciennes troupes de Rigaud et de Louverture rejoignent, en octobre 1802, la rébellion contre la France, Dessalines, en quête d'une identité non européenne, choisit l'Amérique et non l'Afrique comme référence. En novembre 1802, Dessalines établit le siège de son gouvernement à la Petite Rivière de 
l'Artibonite, donne aux populations soumises à son autorité le nom d'Incas et dénomme l'armée indigène, «armée des Incas ». Au qui vive de la sentinelle, le soldat répond: "fils du Soleil, il y en a encore ». Dessalines s'achemine vers le choix du nom indien d'Haïti pour le nouvel État (Geggus 1997 : 43-68). Est-ce uniquement par souci de combattre le parti africain de Sans-Souci qui vient d'être vaincu? Ou bien ce parti a-t-il été évincé juste pour écarter une influence africaine, alors décriée ? L'identité africaine est alors partout rejetée et combattue. Qu'on se rappelle Miranda déclarant préférer le maintien de la domination espagnole plutôt que de voir l'Amérique se transformer en une nouvelle Guinée, comme cela se passe en Haïti (Verna 1980 : 87, 89) ! Dans sa fameuse proclamation du 28 avril 1804, Dessalines déclare : "J'ai vengé l'Amérique ». L'omission de l'Afrique est l'expression d'un silence éloquent. La mise à l'écart de l'Afrique entraîne des conséquences immédiates, en dépit de décisions très louables de Dessalines.

La constitution de 1805 revendique pour le nouvel État une certaine identité africaine en proclamant que l'Haïtien, quelle que soit sa couleur, sera désormais connu sous la dénomination générique de Noir. Dessalines récupère ainsi la mémoire des Indiens et celle de l'Afrique. Bien que la constitution de 1805 prive les Blancs du droit de propriété, elle admet au rang de citoyens haïtiens les Allemands et les Polonais victimes de la politique de Bonaparte. Mais le gouvernement de Dessalines maintient le système militaire de Toussaint. Il divise la population en militaires et en cultivateurs. La constitution de 1805 prive les cultivateurs de la qualité de citoyen en prévoyant que celle-ci suppose la connaissance d'un art mécanique. En vertu de l'ordonnance du 25 octobre 1804, tous ceux qui ne peuvent justifier de leurs moyens d'existence dans les villes doivent être transférés de force à la campagne pour y travailler la terre, sous un strict régime militaire. En même temps, l'élimination du parti de Sans-Souci par Dessalines et Christophe rend plus aisée pour les mulâtres la conquête de la prééminence politique dans le nouvel État. Lorsque Dessalines ordonne la rentrée dans le domaine public de tous les biens des colons partis, les mulâtres déclarent qu'ils leur reviennent comme ayant appartenus à leurs pères. Dessalines, surpris de cette revendication du mulâtre incapable, avant la révolution, de regarder son père blanc dans les yeux, se demande s'il ne restera rien pour les pauvres Noirs dont les pères sont en Afrique. Sa politique ne prouve pas qu'en prononçant ces mots Dessalines ait eu en vue, comme trop souvent on le répète, les pauvres cultivateurs qu'il soumet à la 
glèbe. Ces propos ne traduisent-ils pas de préférence son dépit en découvrant que, finalement, il a été l'Africain des mulâtres?

Toussaint et ses hommes ont voulu éviter à leur personne l'abjection attachée à l'origine africaine. Ils n'y ont pas échappé. Selon Descourtilz,

pendant le règne des noirs la prépondérance était aux Africains. Depuis l'arrivée des Français, ces derniers ont eu l'avantage quelque temps mais dans l'un et l'autre cas, les hommes de couleur servaient toujours de point d'appui pivotant (1809, III : 263).

Descourtilz fait un usage méchant du terme «africain » quand il veut s'en prendre à Toussaint, qu'il considère comme un ennemi. Il se plaît à observer dans le gouverneur le «vieux Africain ", " notre chef africain », «le tyran africain » (245, 258-259). L'Africain n'est pas seulement un méchant; sa conduite est incompréhensible au regard de l'Européen civilisé, le seul être humain doué de toutes les vertus. Le général Pamphile de Lacroix s'étonne de l'art de la guerre chez SansSouci et de la grande capacité d'activité raisonnée chez un être aussi obscur. Acceptant a priori, comme une vérité de foi, la croyance en l'infériorité naturelle des Noirs, Rochambeau, surpris de leur résistance acharnée, informe le ministre Décrès que les Français affrontent à SaintDomingue « une race supérieure d'Africains " (Élie 1945, II : 140). L'Africain ne peut être perçu comme courageux, mais comme féroce et sauvage. Ainsi, le général Capois, quoique créole, est, pour Philippe Albert de Lattre, l'un des Nègres les plus féroces qu'ait produit l'Afrique (Madiou 1989, V : 421). Le parti de Sans-Souci est qualifié d'africain parce qu'il passe pour radical aux yeux d'une minorité apeurée d'indigènes privilégiés.

Le Mulâtre sait également qu'il ne peut s'identifier à l'Européen, qu'il est certainement un Africain aux yeux du Blanc. Mais, une fois celui-ci éliminé, le Mulâtre ne devient-il pas le Blanc local, modelant son discours sur celui des clercs européens ? Madiou dépeint les insurgés dans la plaine du Cul de Sac, au printemps de 1793, comme des bandes effrayantes de Congos, d'Ibos, de Dahomets, de Sénégalais, qu'Alaou, leur chef, domine par la superstition. Il présente les troupes de SansSouci cheminant avec les emblèmes de la superstition de leur pays natal, comme des forces des ténèbres justement vaincues dans l'intérêt national par les forces des lumières représentées par les officiers des anciennes troupes coloniales. Ardouin décrit les insurgés aux ordres d'Alaou comme des bandes indisciplinées qui suivent toutes les pratiques 
« nées de la barbarie de l'Afrique et inconciliables avec la civilisation du peuple » (Ardouin 1958, II : 77). Ces pratiques en font des êtres étranges, bizarres au regard des indigènes natifs de Saint-Domingue, pour lesquels ils sont un objet de honte. Ardouin oppose Christophe, le civilisé, à Sans-Souci, l'Africain barbare, qui ne peut avoir que la force physique et doit, dans son propre intérêt, obéir à la tête dirigeante représentée par les natifs de Saint-Domingue (V:60, 75).

Ces Africains sont un outil pour faire la guerre et un instrument de chantage, un épouvantail pour garantir la liberté des "honnêtes gens". En 1792, Rigaud menace la commission civile de rejoindre les Africains dans leurs retraites escarpées, où il pourrait jouir de la liberté. Le premier janvier 1815, un officier présente à Pétion deux jeunes Haïtiens de sept ans, habillés en paysans des montagnes, le fusil sur le dos. Le Président les embrasse chaleureusement. Relatant l'événement, un journal de l'époque déclare :

Ces petits sauvages sont l'image de notre vie dans les triples montagnes, l'emblème de notre indépendance, la sauvegarde de notre liberté dans le cas que certain peuple civilisé veuille que nous ne le devenions pas (cité par Madiou 1989, V : 276-277).

Mais les plus grands bénéficiaires de cette liberté n'entendent pas être confondus avec l'un de ces sauvages. Une grande crainte de l'épouse et des filles de Christophe, en exil à Londres après le suicide de leur père, roi d'Haïti, en 1820, était d'être prises pour des Africaines (Griggs 1952: 241-245).

\section{L'image de l'Africain}

Avant 1789, quand on parle d'esclave, le terme « africain » demeure sous-entendu. Il surgit au moment de la révolution, comme substitut à esclave. Il désigne le cultivateur, proclamé libre, mais exclu de l'égalité en droit, principalement par les chefs indigènes. Les contrerévolutionnaires en métropole opposent comme incompatibles l'origine africaine et la qualité de citoyen français. De rares républicains, comme Laveaux, pensent fermement au droit des Africains cultivateurs de SaintDomingue de jouir de la qualité de citoyens français. Les chefs mulâtres, tel Rigaud, ne se pensent pas comme Africains et considèrent que seuls les cultivateurs le sont. Les chefs militaires noirs du Nord essaient de faire silence sur leur origine africaine. Ils imposent aux cultivateurs les mêmes rigueurs que leur appliquent les chefs du Sud. Durant la guerre 
d'indépendance en 1802-1803, le terme «africain » prend une connotation de radicalisme politique. Après 1804, le cultivateur demeure la figure de l'Africain transporté d'un territoire lointain pour être destiné à la culture du sol sur lequel il doit être attaché pour le faire fructifier. Ainsi va se perpétuer une certaine image péjorative de l'Africain.

Cette image réelle de l'Africain en Haïti est transfigurée dans le discours officiel et politique qui, assez souvent de façon hypocrite, exalte la race noire. Les clercs se pensent, au plus, comme descendants de l'Afrique et caressent l'espoir que les progrès de la civilisation en Haïti contribueront à la régénération de leur continent d'origine (Légitime 1911 : 26-29). En attendant, ils vivent aux dépens des cultivateurs qui, à leurs yeux, demeurent, avec le vodou, les seuls témoins de l'Afrique en Haïti. Au début du XX $X^{\mathrm{e}}$ siècle, l'école indigéniste en Haïti combattra l'image hideuse de l'Afrique et de l'Africain, et essaiera de la réhabiliter à sa façon et pour ses propres objectifs de pouvoir politique. Mais, au regard de ce qui vient d'être exposé, la reconstruction de l'histoire par les indigénistes est loin d'être scientifique, car il est impossible de faire des leaders noirs, Toussaint, Dessalines et Christophe, des défenseurs de ces cultivateurs que, à l'instar des chefs mulâtres, ils ont impitoyablement opprimés. 


\section{Références}

Alaux, Jean-Paul, 1935, Ulysse aux Antilles. Paris, éditions Histoire. Ardouin, Beaubrun, 1958, Études sur l'histoire d'Haïti (11 vol.). Portau-Prince, Dalencour éditeur.

Barthélemy, Gérard, 1989, «Spécificité, idéologie et rôle des noirs libres pendant la période de l'indépendance d'Haïti ». Dans Michel Hector (dir.), La révolution française et Haïti, tome 1, Port-au-Prince, Deschamps : 169-184.

Bénot, Yves, 1993, «Comment la Convention a-t-elle voté l'abolition de l'esclavage en l'an II ? » Annales Historiques de la Révolution Française 293-294: 349-362.

Courrier de la France et des colonies, 1795, n 1, 17 octobre : 175, 173. Debien, Gabriel, 1974, Les esclaves aux Antilles françaises. Basse-Terre, Société d'histoire de la Guadeloupe.

— 1977, « Toussaint Louverture avant 1789. Légendes et réalités » Conjonction 134 (Port-au-Prince) : 65-80.

-__- 1962, Plantations d'esclaves à Saint-Domingue. Université de Dakar, Publications de la section d'histoire 3.

Duvalier, François et Denis Lorimer, 1968, «Le problème des classes à travers l'histoire d'Haïti ». Dans François Duvalier, Euvres essentielles, tome I, Port-au-Prince, Presses nationales d'Haïti : 314-367.

Descourtilz, Michel Etienne, 1809, Voyages d'un naturaliste (3 vol). Paris, Dufort.

Élie, Louis Émile, 1945, Histoire d'Haïti (2 vol.). Port-au-Prince. Garrigus, John, 1997, «Sons of the Same Father. Gender, Race and Citizenship in French Saint-Domingue, 1760-1792». Dans Christine Adams, Jack R. Censer et Lisa Jane Graham (dir.), Visions and Revisions of the French Eighteenth-Century France, The Pennsylvania State University Press : 137-153.

Gauthier, Florence, 1992, Triomphe et mort du droit naturel en révolution, 1789-1802. Paris, PUF.

Geggus, David, P., 1982, Slavery, War and Revolution. The British Occupation of Santo Domingo, 1793-198. Oxford, Clarendon Press. , 1997a, « Naming of Haiti ». New West Indian Guide 71 (1-2) : 43-68.

-_--, 1997b, «Toussaint Louverture and the Slaves of the Breda Plantation ». Dans Judy Bieber (dir.), Plantation Societies in the Age of European Expansion, 1450-1800, Londres, Ashgate : 266-284.

Griggs, Earl Leslie et Clifford H. Prator, 1952, Henri Christophe and

Thomas Clarkson. A Correspondence. Berkeley, University of California Press. 
Justin, Placide, 1826, Histoire politique et statistique de l'île d'Hayti. Paris, Brière.

Kerverseau, 1939, "Rapport sur la partie française de SaintDomingue ». Boletin del Archivo General de la Nacion 3 : 320-321.

King, Steward R., 2001, Blue Coat or Powdered Whig. Free People of Color in Pre-Revolutionary Saint-Domingue. Athens, The University of Georgia Press.

Lattre, Philippe Albert de, 1805, Campagne des Français à Saint-Domingue et réfutation des reproches faits à Rochambeau. Paris, Locard libraire. Laurent, Gérard, M., 1980, Quand les chaînes volent en éclats. Port-auPrince, Deschamps.

Lefebvre, George, 1950, Robespierre. Oeuvres complètes (vol. 7). Paris, PUF.

Pluchon, Pierre, 1989, Toussaint Louverture, un révolutionnaire noir d'Ancien Régime. Paris, Fayard.

Légitime, François Denis, 1911, La République d'Haïti et les races africaines en général. Congrès universel des races tenu à Londres, 26-29 juillet 1911, Port-au-Prince, L'Abeille.

Le Moniteur, 1795, 29 juillet (11 thermidor an 3).

Logan, Rayford, 1941, The Diplomatic Relations of the United States with Haiti, 1776-1891. Chapel Hill, The University of North Carolina Press.

Madiou, Thomas, 1989, Histoire d'Haïti (8 vol.). Port-au-Prince, H. Deschamps.

Ménier, M. A., 1976, «Toussaint Louverture avant 1789. Légendes et réalités », Conjonction 134 (Port-au-Prince).

Petit, Émilien, 1771, Droit public, ou Gouvernement des colonies françaises d'après les lois faites pour ces pays. Paris, Delain.

— 1777, Traité sur le gouvernement des esclaves. Paris, Knapen.

Price-Mars, Jean, 1925, "Le phénomène religieux chez les nègres de Saint-Domingue ». Revue de la Société d'histoire et de Géographie d'Haïti, 1 (1) : 34-55.

Revue d'histoire des colonies françaises, 1949, «Proclamation de Polvérel du 31 octobre $1793 »$. Tome 36 (127-128) : 372-387.

Saint-Louis, Vertus, 2000, «Les termes citoyen et africain pendant la révolution haïtienne». Dans Laënnec Hurbon (dir.), L'insurrection des esclaves de Saint-Domingue, 22-23 août 1791. Actes de la table ronde internationale de Port-au-Prince, 8 au 10 décembre 1997, Paris, Karthala : 75-95. 
Verna, Paul, 1980, Pétion y Bolivar. Una etapa decisiva en la emancipacion de Hispanoamerica. Caracas, Ediciones de la Presidencia de la Republica.

Wanquet, Claude, 1998, La France et la première abolition de l'esclavage, 1794-1802. Le cas des colonies orientales. Paris, Karthala.

Documents d'archives

\section{Archives nationales de France, fonds des colonies}

Béhague, 1790, «Mémoire sur les colonies de l'Amérique par le comte de Behague, Maréchal des camps, ci-devant commandant en Amérique. Renvoyé au comité des rapports par décret du 2 mars et renvoyé au lundi 8 ». F12 1653.

Brunache, 1794, "Lettre à Polvérel, 7 mars ». D/XXV/25/21/259, doc. 18.

Carnot, Lazare, 1796, «Lettre de Carnot au ministre de la marine du 4 messidor an IV». Gr 132, ancien, 177 bis.

Gavanon, 1794, «Lettre à Polvérel, $1^{\text {er }}$ mars, concernant l'Affricain de l'habitation Charmilly ». D/XXV/25/21/259, doc. 1.

Gavanon, 1794, «Lettre à Polvérel, 3 mars, concernant l'état des journées... ». D/XXV/25/21/259, doc. 2.

Gavanon, 1796, Document de vendémiaire an IV. A.N. 5SUPSDOM5. Kerverseau et Leborgne, 1796, «Rapport fait au gouvernement sur les troubles du... Sud de Saint-Domingue... fructidor an IV». Collection Moreau de Saint-Méry, vol. 68, doc. 2.

Laveaux, Étienne, 1798, «Opinion de Laveaux en la séance du 12 nivôse an VI ». Collection Moreau de Saint-Méry, série 1, vol. 115, $\mathrm{n}^{\circ} 21$.

Laveaux, Étienne, 1794, «Proclamation du 4 mai 1794 ». Collection Moreau de Saint-Méry, série 2, vol. 2, Règlements et proclamations, p. 104.

Laveaux, Étienne, 1794 (22 septembre), «Message à la Convention nationale, $1^{\text {er }}$ vendémiaire an III ». CC9A10.

«Mouvement de la prison de Port-au-Prince », 10 avril 1794. D/XXV/ 26/21/269, document 12 .

Polvérel, Étienne, 1793, «Ordonnance du 24 décembre 1793 concernant une lettre de la municipalité de Port-Salut relative à un soulèvement qui devrait éclater le $1^{\text {er }}$ janvier $1794 »$. D/XXV/10/ $101 / 13$. 
Polvérel, Étienne, 1794, «Lettre datée du Port-Républicain le 16 avril 1794 à Montbrun, commandant de la province de l'Ouest, relative à l'Affricain Zamore ». D/XXV/42.

Rigaud, André, 1797, «Mémoire du général... André Rigaud... 18 thermidor an V". Collection Moreau de Saint-Méry, série 2, vol. 2. Rigaud, André, 1794, «Ordonnance du 25 septembre 1794 ». CC9A9. Rochambeau, 1796, "Extrait de la correspondance du général Rochambeau avec le ministre de la marine. Lettre confidentielle du 11 messidor an IV ». Collection Moreau de Saint-Méry, série 2, volume 2 .

Sonthonax, 1793, «Lettre datée de Port-au-Prince, 13 novembre 1793, à la municipalité de Saint-Marc». D/XXV/43, pp. 41-42.

Bibliothèque des Frères de Saint-Louis, Port-au-Prince

Duboÿs, Pélage-Marie, s.d., Précis historique des annales de la colonie française de Saint-Domingue depuis 1789. Texte dactylographié, 2 vol. 